\title{
ON THE TURBULENT BOUNDARY LAYER OF A DRY GRANULAR AVALANCHE DOWN AN INCLINE. II - CLOSURE MODEL AND NUMERICAL SIMULATIONS
}

\author{
Chung FAng \\ National Cheng Kung University, Department of Civil Engineering, Tainan City, Taiwan \\ e-mail: cfang@mail.ncku.edu.tw
}

\begin{abstract}
Dynamic responses of the closure relations, specific turbulent Helmholtz free energy and turbulent viscosity are postulated followed by experimental calibrations. The established closure model is applied to analyses of a gravity-driven stationary avalanche with incompressible grains down an incline. While the mean velocity and volume fraction increase from their minimum values on the plane toward maximum values on the free surface exponentially, two-fold turbulent kinetic energies and dissipations evolve in a reverse manner. Most two-fold turbulent kinetic energies and dissipations are confined within the thin turbulent boundary layer immediately above the plane, with nearly vanishing two-fold turbulent kinetic energies and finite two-fold turbulent dissipations in the passive layer. The two layers are similar to those of Newtonian fluids in turbulent boundary layer flows, and are preferable recognized by the distributions of turbulent kinetic energies and dissipations.
\end{abstract}

Keywords: closure model, gravity-driven flow, passive layer, turbulent boundary layer

\section{Introduction}

This paper continues Fang (2016b), hereafter referred to as Part I. The balance equations of the mean fields for isothermal flows with incompressible grains are summarized in the following

$$
\begin{array}{ll}
0=\dot{\bar{\nu}}+\bar{\nu} \nabla \cdot \overline{\mathbf{v}} & \mathbf{0}=\bar{\gamma} \overline{\bar{\nu}} \dot{\mathbf{v}}-\operatorname{div}(\overline{\mathbf{t}}+\mathbf{R})-\bar{\gamma} \bar{\nu} \overline{\mathbf{b}} \\
0=\bar{\gamma} \bar{\nu} \ell \ddot{\bar{\nu}}-\nabla \cdot(\overline{\mathbf{h}}+\mathbf{H})-\bar{\gamma} \bar{\nu} \bar{f} & \mathbf{0}=\overline{\overline{\mathbf{Z}}}-\overline{\mathbf{\Phi}} \quad(\overline{\mathbf{Z}} \equiv \dot{\overline{\mathbf{Z}}}-[\overline{\mathbf{\Omega}}, \overline{\mathbf{Z}}]) \\
0=\bar{\gamma} \bar{\nu} \dot{k}-\mathbf{R} \cdot \overline{\mathbf{D}}-\nabla \cdot \mathbf{K}+\bar{\gamma} \bar{\nu} \varepsilon & 0=\bar{\gamma} \bar{\nu} \dot{s}-\ell \mathbf{H} \cdot \nabla \dot{\bar{\nu}}-\nabla \cdot \mathbf{L}+\bar{\gamma} \bar{\nu} H
\end{array}
$$

for which

$$
\mathcal{P}=\left\{\bar{p}, \bar{\nu}, \overline{\mathbf{v}}, \overline{\mathbf{Z}}, \vartheta^{M}, \vartheta^{T}, \vartheta^{G}\right\} \quad \mathcal{C}=\{\overline{\mathbf{t}}, \mathbf{R}, \overline{\mathbf{h}}, \mathbf{H}, \bar{f}, \overline{\mathbf{\Phi}}, k, s, \mathbf{K}, \mathbf{L}, \varepsilon, H\}
$$

are introduced respectively as the primitive mean fields and closure relations based on the turbulent state space given by

$$
\mathcal{Q}=\left\{\nu_{0}, \bar{\nu}, \dot{\bar{\nu}}, \mathbf{g}_{1}, \bar{\gamma}=c_{1}, \mathbf{g}_{2}, \vartheta^{M}=c_{2}, \mathbf{g}_{3}, \vartheta^{T}, \mathbf{g}_{4}, \vartheta^{G}, \mathbf{g}_{5}, \overline{\mathbf{D}}, \overline{\mathbf{Z}}\right\} \quad \mathcal{C}=\hat{\mathcal{C}}(\mathcal{Q})
$$

with $c_{1}$ and $c_{2}$ are constants, and $\mathbf{g}_{2}=\mathbf{g}_{3}=\mathbf{0}$. Quantities in (1.1)-(1.3) have been defined in Part I. Müller-Liu entropy principle has been investigated to derive the equilibrium closure relations, with the results summarized in Table 1, in which the subscript $E$ denotes that the indexed quantity is evaluated at an equilibrium state, defined viz.,

$$
\left.\mathcal{Q}\right|_{E} \equiv\left(\nu_{0}, \bar{\nu}, 0, \mathbf{g}_{1}, c_{1}, \mathbf{0}, c_{2}, \mathbf{0}, \vartheta^{T}, \mathbf{0}, \vartheta^{G}, \mathbf{0}, \mathbf{0}, \overline{\mathbf{Z}}\right) \quad \mathcal{Q}^{D} \equiv\left(\dot{\bar{\nu}}, \mathbf{g}_{3}, \mathbf{g}_{4}, \mathbf{g}_{5}, \overline{\mathbf{D}}\right)
$$

with $\mathcal{Q}^{D}$ the dynamic sub-state space, upon which the dynamic closure relations should depend. 
Table 1. Thermodynamically consistent equilibrium closure relations (Fang, 2016b)

$$
\begin{array}{llc}
\psi^{T}=\hat{\psi}^{T}\left(\nu_{0}, \bar{\nu}, \nabla \bar{\nu}, \bar{\gamma}=c_{1}, \vartheta^{M}=c_{2}, \vartheta^{T}, \vartheta^{G}, \overline{\mathbf{Z}}\right) & \bar{\beta}=\bar{\gamma} \bar{\nu} \psi_{\bar{\nu}}^{T} \\
\bar{\gamma} \bar{\nu} k=\bar{\gamma} \bar{\nu} \vartheta^{M} \psi_{, \vartheta^{T}}^{T} & \bar{\gamma} \bar{\nu} s=\bar{\gamma} \bar{\nu} \vartheta^{M} \psi_{, \vartheta}^{T} & \\
\left.\bar{\gamma} \bar{\nu} \varepsilon\right|_{E}=0 & \left.\bar{\gamma} \bar{\nu} H\right|_{E}=0 & \left.\mathbf{\boldsymbol { \Phi }}\right|_{E}=\mathbf{0} \\
\left.\mathbf{K}\right|_{E}=\left.\left(\vartheta^{M}-\vartheta^{T}\right) \bar{\gamma} \bar{\nu} \varepsilon_{\mathbf{g}_{4}}\right|_{E}+\left.\left(\vartheta^{M}-\vartheta^{G}\right) \bar{\gamma} \bar{\nu} H_{\mathbf{g}_{4}}\right|_{E}-\left.\bar{\gamma} \bar{\nu} \vartheta^{M} \psi_{, \overline{\mathbf{Z}}}^{T} \cdot \overline{\mathbf{\Phi}}_{, \mathbf{g}_{4}}\right|_{E} \\
\left.\mathbf{L}\right|_{E}=\left.\left(\vartheta^{M}-\vartheta^{T}\right) \bar{\gamma} \bar{\nu} \varepsilon_{, \mathbf{g}_{5}}\right|_{E}+\left.\left(\vartheta^{M}-\vartheta^{G}\right) \bar{\gamma} \bar{\nu} H_{, \mathbf{g}_{5}}\right|_{E}-\left.\bar{\gamma} \bar{\nu} \vartheta^{M} \psi_{, \overline{\mathbf{Z}}}^{T} \cdot \overline{\mathbf{\Phi}}_{, \mathbf{g}_{5}}\right|_{E} \\
\ell\left(\vartheta^{M} \overline{\mathbf{h}}+\vartheta^{G} \mathbf{H}\right)=\bar{\gamma} \bar{\nu} \vartheta^{M} \psi_{, \mathbf{g}_{1}}^{T} \quad \mathbf{H}=\ell \mathbf{R g}_{1} \\
\left.\bar{f}\right|_{E}=(\ell)^{-1}\left\{(\bar{p}-\bar{\beta}) /(\bar{\gamma} \bar{\nu})+\left.\left(1-\vartheta^{T} / \vartheta^{M}\right) \varepsilon_{, \dot{\nu}}\right|_{E}+\left.\left(1-\vartheta^{G} / \vartheta^{M}\right) H_{, \dot{\nu}}\right|_{E}-\left.\psi_{, \overline{\mathbf{Z}}}^{T} \cdot \overline{\mathbf{\Phi}}_{, \dot{\nu}}\right|_{E}\right\} \\
\left.\overline{\mathbf{t}}\right|_{E}=-\bar{\nu} \overline{\mathbf{I}}-\bar{\gamma} \bar{\nu} \psi_{, \mathbf{g}_{1}}^{T} \otimes \mathbf{g}_{1}+\bar{\gamma} \bar{\nu} \psi_{, \overline{\mathbf{Z}}}^{T} \cdot \overline{\mathbf{\Phi}}_{,\left.\overline{\mathbf{D}}\right|_{E}} \\
\left.\mathbf{R}\right|_{E}=-\left.\left(\vartheta^{M} / \vartheta^{T}-1\right) \bar{\gamma} \bar{\nu} \varepsilon_{, \mathbf{D}}\right|_{E}-\left.\left(\vartheta^{M} / \vartheta^{T}-\vartheta^{G} / \vartheta^{T}\right) \bar{\gamma} \bar{\nu} H_{, \mathbf{D}}\right|_{E} \\
\hline
\end{array}
$$

In Section 2, the dynamic responses of the closure relations are postulated by a quasi-static theory, followed by the specific postulates of the turbulent Helmholtz free energy, viscosities and the hypoplastic model for rate-independent characteristics. The established closure model is applied to analyses of a gravity-driven stationary avalanche down an incline in Section 3. Numerical simulations are compared with laminar flow solutions. The study is concluded in Section 4.

\section{Zero-order closure model}

\subsection{Dynamic response}

It is assumed that the closure relations consist of the equilibrium and dynamic parts viz.

$$
\mathcal{C}=\left.\mathcal{C}\right|_{E}+\mathcal{C}^{D} \quad \mathcal{C} \in\{\overline{\mathbf{t}}, \mathbf{R}, \overline{\mathbf{\Phi}}, \mathbf{K}, \mathbf{L}, \bar{f}, \bar{\gamma} \bar{\nu} \varepsilon, \bar{\gamma} \bar{\nu} H\}
$$

Specifically, $\overline{\mathbf{t}}^{D}, \mathbf{R}^{D}, \bar{f}^{D}, \bar{\gamma} \bar{\nu} \varepsilon^{D}, \bar{\gamma} \bar{\nu} H^{D}, \mathbf{K}^{D}$ and $\mathbf{L}^{D}$ are assumed to be the quasi-static expressions of $\mathcal{Q}^{D}$ given by

$$
\begin{array}{ll}
\mathbf{0}=\overline{\mathbf{t}}^{D}-\epsilon^{M} \dot{\bar{\nu}} \mathbf{I}-\lambda^{M}(\operatorname{tr} \overline{\mathbf{D}}) \mathbf{I}-2 \mu^{M} \overline{\mathbf{D}} & \mathbf{0}=\mathbf{R}^{D}-\epsilon^{T} \dot{\bar{\nu}} \mathbf{I}-\lambda^{T}(\operatorname{tr} \overline{\mathbf{D}}) \mathbf{I}-2 \mu^{T} \overline{\mathbf{D}} \\
0=f^{D}+\zeta \dot{\bar{\nu}}+\delta(\operatorname{tr} \overline{\mathbf{D}}) & 0=\bar{\gamma} \bar{\nu} \varepsilon^{D}-f_{1} \dot{\bar{\nu}}-f_{2}(\operatorname{tr} \overline{\mathbf{D}})-f_{3}\left(\mathbf{g}_{4} \cdot \mathbf{g}_{4}\right) \\
0=\bar{\gamma} \bar{\nu} H^{D}-f_{4} \dot{\bar{\nu}}-f_{5}(\operatorname{tr} \overline{\mathbf{D}})-f_{6}\left(\mathbf{g}_{5} \cdot \mathbf{g}_{5}\right) & \mathbf{0}=\mathbf{K}^{D}+f_{7} \mathbf{g}_{4} \quad \mathbf{0}=\mathbf{L}^{D}+f_{8} \mathbf{g}_{5}
\end{array}
$$

with $\epsilon^{M}, \epsilon^{T}, \zeta, f_{1}, f_{3}, f_{4}, f_{6-8}$ being scalar functions of $\left(\nu_{0}, \bar{\nu}, \bar{\gamma}, \vartheta^{M}, \vartheta^{T}, \vartheta^{G}\right)$; and $\lambda^{M}, \lambda^{T}, \delta$, $\mu^{M}, \mu^{T}, f_{2}, f_{5}$ scalar functions depending additionally on the three invariants $\left(I_{\overline{\mathbf{D}}}^{1}, I_{\overline{\mathbf{D}}}^{2}, I_{\overline{\mathbf{D}}}^{3}\right)$ of $\overline{\mathbf{D}}$ given by $I_{\overline{\mathbf{D}}}^{1} \equiv \operatorname{tr} \overline{\mathbf{D}}, I_{\overline{\mathbf{D}}}^{2} \equiv 0.5\left(\operatorname{tr}^{2} \overline{\mathbf{D}}-\operatorname{tr} \overline{\mathbf{D}}^{2}\right)$ and $I_{\overline{\mathbf{D}}}^{3} \equiv \operatorname{det} \overline{\mathbf{D}}$.

In equation (2.2), Truesdell's equi-presence principle is used, by which $\left(\vartheta^{M} \overline{\mathbf{t}}^{D}+\vartheta^{T} \mathbf{R}^{D}\right)$ and $\bar{f}^{D}$ depend explicitly and linearly on $\dot{\bar{\nu}}$ and $\overline{\mathbf{D}} ; \bar{\gamma} \bar{\nu} \varepsilon^{D}$ and $\bar{\gamma} \bar{\nu} H^{D}$ depend explicitly and linearly on $\dot{\bar{\nu}}, \overline{\mathbf{D}}, \mathbf{g}_{4}$ and $\mathbf{g}_{5} ; \mathbf{K}^{D}$ and $\mathbf{L}^{D}$ depend explicitly and linearly on $\mathbf{g}_{4}$ and $\mathbf{g}_{5}$, respectively, motivated by the Fourier law. Thus, a dry granular avalanche is considered a Stokes or Reiner-Rivlin fluid. Scalar functions $\mu^{M}$ and $\mu^{T}$ are respectively the material viscosity and phenomenological (turbulent) viscosity induced by turbulent fluctuation. Equation (2.2) has been applied for creeping, dense and rapid laminar flows, and for weak turbulent dense flows as the first approximation (Fang, 2008, 2009, 2016a; Fang and Wu, 2014; Kirchner and Teufel, 2002; Wang and Hutter, 1999). 


\subsection{Turbulent Helmholtz free energy, material and turbulent viscosities}

It is assumed that $\psi^{T}$ consists of an elastic part, $\psi_{e}^{T}$, and a rate-independent part, $\psi_{f}^{T}$, viz.

$$
\psi^{T}=\psi_{e}^{T}\left(\nu_{0}, \bar{\nu}, \mathbf{g}_{1}, \bar{\gamma}, \vartheta^{M}, \vartheta^{T}, \vartheta^{G}\right)+\psi_{f}^{T}\left(I_{\overline{\mathbf{Z}}}^{1}, I_{\overline{\mathbf{Z}}}^{2}, I_{\overline{\mathbf{Z}}}^{3}\right)
$$

with the irreversible effect confined within $\psi_{f}^{T}$ (Fang, 2009; Kirchner and Teufel, 2002; Wang and Hutter, 1999). Following the previous works, $\psi_{e}^{T}$ is assumed to be expanded in a Taylor series about $\bar{\nu}=\bar{\nu}_{m}$ and $\left|\mathbf{g}_{1}\right|=0$, with $\bar{\nu}_{m}$ the critical mean volume fraction at which shearing is decoupled from dilatation, see Fang (2009), Savage (1993), Wang and Hutter (2001)

$$
\begin{aligned}
& \psi_{e}^{T}=\left\{\alpha_{0}\left(\bar{\nu}-\bar{\nu}_{m}\right)^{2}+\beta_{0}\left(\frac{\bar{\nu}_{m}}{\bar{\nu}_{\infty}-\bar{\nu}}\right)^{2} \mathbf{g}_{1} \cdot \mathbf{g}_{1}\right\} \mathcal{F}_{c} \\
& \mathcal{F}_{c}=\sum_{n=0}^{n=2} \frac{1}{n !}\left\{\left(\frac{\vartheta^{T}}{\vartheta^{M}}\right)^{n}+\left(\frac{\vartheta^{G}}{\vartheta^{M}}\right)^{n}\right\}-1
\end{aligned}
$$

with $\bar{\nu}_{\infty}$ the value of $\bar{\nu}$ corresponding to the denst possible packing of the grains, and $\left\{\alpha_{0}, \beta_{0}\right\}$ depending on $\left\{\bar{\nu}_{m}, \bar{\gamma}, \vartheta^{M}\right\}$. Equation (2.4) is an extension of its laminar flow counterpart with $\mathcal{F}_{c}$ accounting for the influence of turbulent fluctuation, motivated by the nonlinear characteristics of rapid flows (Pudasaini and Hutter, 2007; Rao and Nott, 2008; Wang and Hutter, 2001). It asserts that smaller two-fold granular coldnesses result in smaller free energy.

The specific forms of the material viscosity $\mu^{M}$ and turbulent viscosity $\mu^{T}$ are given by

$$
\mu^{M}=\mu_{0} \bar{\gamma}^{2}\left(\frac{\bar{\nu}_{m}}{\bar{\nu}_{\infty}-\bar{\nu}}\right)^{8} \Xi \quad \mu^{T}=\mu_{0} \bar{\gamma}^{2}\left(\mathcal{F}_{c}-1\right)\left(\frac{\bar{\nu}_{m}}{\bar{\nu}_{\infty}-\bar{\nu}}\right)^{8} \Xi
$$

with $\Xi=\hat{\Xi}\left(I_{\overline{\mathbf{D}}}^{1}, I_{\overline{\mathbf{D}}}^{2}, I_{\overline{\mathbf{D}}}^{3}\right)$, and $\mu_{0}=\hat{\mu}_{0}\left(\nu_{0}, \vartheta^{M}\right)$, a positive constant. They are postulated followed by the previous works (Fang, 2009; Kirchner and Teufel, 2002), with the power 8 a curve-fitting (Savage, 1993), and the dependency of $\mu^{T}$ on $\vartheta^{T}$ and $\vartheta^{G}$ motivated by Newtonian fluids in turbulent flow. Both $\mu^{M}$ and $\mu^{T}$ assert that total stress is larger in turbulent flows than in laminar flows. For laminar flows, both $\vartheta^{T}$ and $\vartheta^{G}$ vanish, yielding the vanishing $\mu^{T}$.

\subsection{Hypoplasticity}

A hypoplastic model of $\overline{\boldsymbol{\Phi}}$ is given by (Fellin, 2013; Fuentes et al., 2012; Niemunis et al., 2009)

$$
\overline{\mathbf{\Phi}}=\hat{\overline{\mathbf{\Phi}}}(\bar{\nu}, \overline{\mathbf{D}}, \overline{\mathbf{Z}})=f_{s}\left(\bar{\nu}, I_{\bar{Z}}^{1}\right)\left\{a^{2} \overline{\mathbf{D}}+\check{\overline{\mathbf{Z}}} \operatorname{tr}(\check{\overline{\mathbf{Z}}} \overline{\mathbf{D}})+f_{d}(\bar{\nu}) a\left(\check{\overline{\mathbf{Z}}}+\check{\mathbf{Z}}^{*}\right)\|\overline{\mathbf{D}}\|\right\}
$$

for rate-independent characteristics, with $\check{\overline{\mathbf{Z}}}=\overline{\mathbf{Z}} / \operatorname{tr}(\overline{\mathbf{Z}})$, the versor of $\overline{\mathbf{Z}} ; \check{\overline{\mathbf{Z}}}^{*}=\check{\overline{\mathbf{Z}}}-\mathbf{I} / 3$, the deviator of $\overline{\mathbf{Z}} ;\|\overline{\mathbf{D}}\|=\sqrt{\operatorname{tr} \overline{\mathbf{D}}^{2}}$; and $a$ a positive constant. The scalar functions $f_{s}$ and $f_{d}$ are respectively the stiffness and density factors. The constant $a$ is related to the stress state $\overline{\mathbf{Z}}_{c}$ and frictional angle $\varphi_{c}$ in the critical state, and can be determined empirically (Bauer and Herle, 2000; Buscamera, 2014; Marcher et al., 2000). Equation (2.6) is used to account for the rate-independent features of dry granular systems, with the benefits that (1) distinction between loading and unloading is automatically accomplished, and (2) elastic/inelastic deformations need not a priori be distinguished; information about yield surface and plastic potential is no longer necessary. 


\subsection{Closure relations}

With these, the closure relations of an isochoric flow are given by

$$
\begin{aligned}
& 0=\bar{\gamma} \bar{\nu} k-\bar{\gamma} \bar{\nu}\left[\alpha_{0}\left(\bar{\nu}-\bar{\nu}_{m}\right)^{2}+\beta_{0}\left(\frac{\bar{\nu}_{m}}{\bar{\nu}_{\infty}-\bar{\nu}}\right)^{2}\left(\mathbf{g}_{1} \cdot \mathbf{g}_{1}\right)\right]\left(1+\frac{\vartheta^{T}}{\vartheta^{M}}\right) \\
& 0=\bar{\gamma} \bar{\nu} \varepsilon-f_{1} \dot{\bar{\nu}}-f_{2}(\operatorname{tr} \overline{\mathbf{D}})-f_{3}\left(\mathbf{g}_{4} \cdot \mathbf{g}_{4}\right) \\
& 0=\bar{\gamma} \bar{\nu} H-f_{4} \dot{\bar{\nu}}-f_{5}(\operatorname{tr} \overline{\mathbf{D}})-f_{6}\left(\mathbf{g}_{5} \cdot \mathbf{g}_{5}\right) \\
& 0=\bar{\gamma} \bar{\nu} s-\bar{\gamma} \bar{\nu}\left[\alpha_{0}\left(\bar{\nu}-\bar{\nu}_{m}\right)^{2}+\beta_{0}\left(\frac{\bar{\nu}_{m}}{\bar{\nu}_{\infty}-\bar{\nu}}\right)^{2}\left(\mathbf{g}_{1} \cdot \mathbf{g}_{1}\right)\right]\left(1+\frac{\vartheta^{G}}{\vartheta^{M}}\right) \\
& \mathbf{0}=\ell\left(\vartheta^{M} \overline{\mathbf{h}}+\vartheta^{G} \mathbf{H}\right)-2 \beta_{0} \bar{\gamma} \bar{\nu} \vartheta^{M} \mathcal{F}_{c}\left(\frac{\bar{\nu}_{m}}{\bar{\nu}_{\infty}-\bar{\nu}}\right)^{2} \mathbf{g}_{1} \\
& \mathbf{0}=\mathbf{K}+f_{7} \mathbf{g}_{4} \quad \mathbf{0}=\mathbf{L}+f_{8} \mathbf{g}_{5} \\
& 0=\bar{f}-\frac{\bar{p}}{\bar{\gamma} \bar{\nu} \ell}+\frac{2}{\ell}\left[\alpha_{0}\left(\bar{\nu}-\bar{\nu}_{m}\right)+\frac{\beta_{0} \bar{\nu}_{m}^{2}}{\left(\bar{\nu}_{\infty}-\bar{\nu}\right)^{3}}\left(\mathbf{g}_{1} \cdot \mathbf{g}_{1}\right)\right] \mathcal{F}_{c}-\left(1-\frac{\vartheta^{T}}{\vartheta^{M}}\right) \frac{f_{1}}{\bar{\gamma} \bar{\nu} \ell} \\
& -\left(1-\frac{\vartheta^{G}}{\vartheta^{M}}\right) \frac{f_{4}}{\bar{\gamma} \bar{\nu} \ell}+\zeta \dot{\bar{\nu}}+\delta(\operatorname{tr} \overline{\mathbf{D}}) \\
& \mathbf{0}=\overline{\mathbf{t}}-\left(-\bar{\nu} p+\epsilon^{M} \dot{\bar{\nu}}+\lambda^{M} \operatorname{tr} \overline{\mathbf{D}}\right) \mathbf{I}-f_{s}\left(\zeta_{1} \mathbf{I}+\zeta_{2} \overline{\mathbf{Z}}+\zeta_{3} \overline{\mathbf{Z}}^{2}\right) \\
& +2 \beta_{0} \bar{\gamma} \bar{\nu} \mathcal{F}_{c}\left(\frac{\bar{\nu}_{m}}{\bar{\nu}_{\infty}-\bar{\nu}}\right)^{2} \mathbf{g}_{1} \otimes \mathbf{g}_{1}-2 \mu_{0} \bar{\gamma}^{2}\left(\frac{\bar{\nu}_{m}}{\bar{\nu}_{\infty}-\bar{\nu}}\right)^{8} \sqrt{\left|I_{\mathbf{D}}^{2}\right|} \overline{\mathbf{D}} \\
& \mathbf{0}=\mathbf{R}-\left[-\left(\frac{\vartheta^{M}}{\vartheta^{T}}-1\right) f_{2}-\left(\frac{\vartheta^{M}}{\vartheta^{T}}-\frac{\vartheta^{G}}{\vartheta^{T}}\right) f_{5}+\epsilon^{T} \dot{\bar{\nu}}+\lambda^{T} \operatorname{tr} \overline{\mathbf{D}}\right] \mathbf{I} \\
& -2 \mu_{0} \bar{\gamma}^{2}\left(\mathcal{F}_{c}-1\right)\left(\frac{\bar{\nu}_{m}}{\bar{\nu}_{\infty}-\bar{\nu}}\right)^{8} \sqrt{\left|I_{\overline{\mathbf{D}}}^{2}\right|} \overline{\mathbf{D}}
\end{aligned}
$$

where the Cayley-Hamilton theorem and the notations

$$
\begin{array}{lcc}
c_{1}=\psi_{f, I_{\overline{\mathbf{Z}}}^{1}}^{T} & c_{2}=\psi_{f, I_{\overline{\mathbf{Z}}}^{2}}^{T} & c_{3}=\psi_{f, I_{\overline{\mathbf{Z}}}^{3}}^{T} \\
\left(I_{\overline{\mathbf{Z}}}^{1}\right)_{, \overline{\mathbf{Z}}}=\mathbf{I} & \left(I_{\overline{\mathbf{Z}}}^{2}\right)_{, \overline{\mathbf{Z}}}=I_{\overline{\mathbf{Z}}}^{1} \mathbf{I}-\overline{\mathbf{Z}} & \left(I_{\overline{\mathbf{Z}}}^{3}\right)_{, \overline{\mathbf{Z}}}=I_{\overline{\mathbf{Z}}}^{3} \overline{\mathbf{Z}}^{-1} \\
\zeta_{1}=a^{2}\left(c_{1}+c_{2} I_{\overline{\mathbf{Z}}}^{1}\right)-c_{3} a^{2} I_{\overline{\mathbf{Z}}}^{2} I_{\overline{\mathbf{Z}}}^{3} & \zeta_{3}=c_{3} a^{2}\left(I_{\overline{\mathbf{Z}}}^{3}\right)^{2} \\
\zeta_{2}=\left(c_{1}+c_{2} I_{\overline{\mathbf{Z}}}^{1}\right)\left(I_{\overline{\mathbf{Z}}}^{1}\right)^{-1}-c_{2}\left(a^{2}+\left(I_{\overline{\mathbf{Z}}}^{1}\right)^{-2} \operatorname{tr} \overline{\mathbf{Z}}^{2}\right)+c_{3} I_{\overline{\mathbf{Z}}}^{3}\left(3\left(I_{\overline{\mathbf{Z}}}^{1}\right)^{-2}+a^{2} I_{\overline{\mathbf{Z}}}^{1}\right)
\end{array}
$$

have been used. For laminar flows, (2.7) reduce to those in previous work (Fang, 2009). Two-fold turbulent kinetic energies in $(2.7)_{1}$ and $(2.7)_{4}$ are determined once $\bar{\nu}$ is known.

The field equations of $\left\{\bar{p}, \overline{\mathbf{v}}, \bar{\nu}, \vartheta^{T}, \vartheta^{G}, \overline{\mathbf{Z}}\right\}$ are obtained by substituting (2.7) into (1.1). Since the system is mathematically likely well-posed, one has the chance to obtain the primitive mean fields. In applying (2.7), the phenomenological parameters $\alpha_{0}, \beta_{0}, f_{1-8}, f_{s}, f_{d}, a, \epsilon^{M}, \lambda^{M}, \epsilon^{T}$, $\lambda^{T}, \mu_{0}, \zeta_{1-3}, \bar{\nu}_{m}$ and $\bar{\nu}_{\infty}$ need be prescribed. Since detailed information of them is insufficient, numerical simulation is restricted to a parametric study.

\section{Gravity-driven flow}

\subsection{Field equations and boundary conditions}

Consider a fully-developed, isochoric, two-dimensional stationary avalanche down an incline, as shown in Fig. 1. It is assumed that

$$
\begin{array}{lll}
\overline{\mathbf{v}}=[\bar{u}(y), \bar{v}(y), 0] & \bar{\nu}=\bar{\nu}(y) & \bar{p}=\bar{p}(y) \\
\vartheta^{T}=\vartheta^{T}(y) & \vartheta^{G}=\vartheta^{G}(y) & \bar{Z}_{i j}=\bar{Z}_{i j}(y)
\end{array}
$$


with $\bar{v} / \bar{u} \sim 0 ; u^{\prime} \neq 0, v^{\prime} \neq 0 ;\{i, j\}=(x, y)$, motivated by the assumptions that $\alpha_{, x} \ll \alpha_{, y}$ for any quantity $\alpha$ in simple turbulent shear flows of Newtonian fluids (Batchelor, 1993). The flow corresponds to the critical state defined as the state in which $\dot{\bar{\rho}}=0$ and $\stackrel{\circ}{\mathbf{Z}}=\mathbf{0}$ (Ai et al., 2014; Kirchner and Teufel, 2002). Since in the critical state $f_{d}$ is set to be unity, equations $(1.1)_{4}$ and (2.6) reduce to

$$
\mathbf{0}=f_{s}\left\{a_{c}^{2} \overline{\mathbf{D}}+\check{\overline{\mathbf{Z}}} \operatorname{tr}(\check{\mathbf{Z}} \overline{\mathbf{D}})+a_{c}\left(\check{\mathbf{Z}}^{\prime}+\check{\mathbf{Z}}^{*}\right)\|\overline{\mathbf{D}}\|\right\}
$$

with $a_{c}=\sqrt{8 / 27} \sin \varphi_{c}$, and $\varphi_{c}$ the critical friction angle (Kirchner and Teufel, 2002). Since $f_{s}$ does not vanish generally, substituting (3.1) into (3.2) yields

$$
\begin{array}{ll}
0 & =\check{\bar{Z}}_{x x} \check{\bar{Z}}_{x y} m+a_{c}\left(2 \check{\bar{Z}}_{x x}-\frac{1}{3}\right) \quad 0=\check{\bar{Z}}_{y y} \check{\bar{Z}}_{x y} m+a_{c}\left(2 \check{\bar{Z}}_{y y}-\frac{1}{3}\right) \\
0 & =a_{c}^{2} m+\check{Z}_{x y}^{2} m+2 a_{c} \check{\bar{Z}}_{x y}
\end{array}
$$

with $m \equiv \bar{D}_{x y} /\|\overline{\mathbf{D}}\|=\bar{D}_{x y} /\left|\bar{D}_{x y}\right|$. A non-trivial solution to (3.3) is only that $\bar{Z}_{x x}=\bar{Z}_{y y}$ and $\bar{Z}_{x y}=-m \sqrt{8 / 3} \sin \varphi_{c} \bar{Z}_{y y}$. Thus, equation (1.1) $)_{4}$ is decoupled from other mean balance equations. For further identification, a specific form of $f_{s}$ is given by (Bauer and Herle, 2000)

$$
f_{s}=\left(\frac{1-\bar{\nu}_{s}}{1-\bar{\nu}}\right)^{m} \quad m=1
$$

with $\bar{\nu}_{s}$ the minimum mean volume fraction; and unity power justified for most cases (Herle and Gudehus, 2000; Marcher et al., 2000; Niemunis et al., 2009).

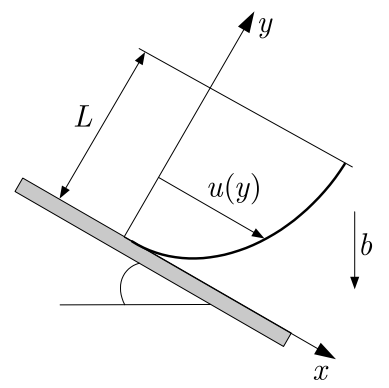

Fig. 1. Gravity-driven stationary avalanche down an incline and the coordinate

With these, the field equations are obtained

$$
\begin{aligned}
0= & \frac{d}{d y}\left\{\frac{1-\bar{\nu}_{s}}{1-\bar{\nu}}\left(\zeta_{2} \bar{Z}_{x y}+\zeta_{3} \bar{Z}_{x y}^{2}\right)+\mu_{0} \bar{\gamma}^{2} \mathcal{F}_{c}\left(\frac{\bar{\nu}_{m}}{\bar{\nu}_{\infty}-\bar{\nu}}\right)^{8}\left(\frac{d \bar{u}}{d y}\right)^{2}\right\}+\bar{\gamma} \bar{\nu} \sin \theta \\
0= & \frac{d \bar{t}_{y y}}{d y}-\bar{\gamma} \bar{\nu} \cos \theta \\
0= & \frac{d}{d y}\left\{\frac{2 \beta_{0} \bar{\gamma} \bar{\nu} \mathcal{F}_{c}}{\ell}\left(\frac{\bar{\nu}_{m}}{\bar{\nu}_{\infty}-\bar{\nu}}\right)^{2} \frac{d \bar{\nu}}{d y}\right\}+\frac{1}{\bar{\nu} \ell}\left\{-\bar{t}_{y y}+\frac{1-\bar{\nu}_{s}}{1-\bar{\nu}} l\left(\zeta_{1}+\zeta_{2} \bar{Z}_{y y}+\zeta_{3} \bar{Z}_{y y}^{2}\right)\right. \\
& \left.-2 \alpha_{0} \bar{\gamma}^{2}\left(\bar{\nu}-\bar{\nu}_{m}\right) \mathcal{F}_{c}-2 \beta_{0} \bar{\gamma} \bar{\nu}\left(\frac{\bar{\nu}_{m}}{\bar{\nu}_{\infty}-\bar{\nu}}\right)^{2}\left(\frac{d \bar{\nu}}{d y}\right)^{2} \frac{\bar{\nu}_{\infty} \mathcal{F}_{c}}{\bar{\nu}_{\infty}-\bar{\nu}}\right\} \\
0= & \mu_{0} \bar{\gamma}^{2}\left(\mathcal{F}_{c}-1\right)\left(\frac{\bar{\nu}_{m}}{\bar{\nu}_{\infty}-\bar{\nu}}\right)^{8}\left(\frac{d \bar{u}}{d y}\right)^{3}-f_{7} \frac{d^{2} \vartheta^{T}}{d y^{2}}-f_{3}\left(\frac{d \vartheta^{T}}{d y}\right)^{2} \\
0= & -f_{8} \frac{d^{2} \vartheta^{G}}{d y^{2}}-f_{6}\left(\frac{d \vartheta^{G}}{d y}\right)^{2}
\end{aligned}
$$

for $\bar{u}(y), \bar{\nu}(y), \bar{t}_{y y}(y), \vartheta^{T}(y)$ and $\vartheta^{G}(y)$, in which $\bar{p}(y)$ is replaced by $\bar{t}_{y y}(y)$ for simplicity. 
Although solid boundaries have been demonstrated to act as energy sources and sinks of the turbulent kinetic energy of the grains (e.g. Pudasaini and Hutter, 2007; Richman and Marciniec, 1990), and the conventional no-slip condition for $\bar{u}$ does not hold (Savage, 1993). The field observations suggest that the no-slip condition can still be used as the first approximation (Pudasaini and Hutter, 2007; Rao and Nott, 2008), with which a fixed value of $\bar{\nu}$ is assumed. As motivated by the finite turbulent kinetic energies and dissipations on solid boundaries of Newtonian fluids in turbulent boundary layer flows (Batchelor, 1993; Tsinober, 2009), finite two-fold turbulent kinetic energies and dissipations on the plane are assumed through the prescriptions of $\vartheta^{T}$ and $\vartheta^{G}$, respectively. On the other hand, field observations suggest that the grains on the free surface interlock with one another to form a kind of inelastic network. Due to a significant density difference between the grains and the air, the shear stress is negligible, yielding vanishing normal gradients of $\bar{u}$ and $\bar{\nu}$. However, the air entrainment on the free surface provides a finite normal stress, giving a rise to nonvanishing $\bar{t}_{y y}$ and normal gradients of $\vartheta^{T}$ and $\vartheta^{G}$ (Pudasaini and Hutter, 2007; Rao and Nott, 2008; Wang and Hutter, 2001). Thus, BCs for (3.5) are postulated by:

- for $y=0$

$$
\bar{u}=0 \quad \bar{\nu}=\bar{\nu}_{b} \quad \vartheta^{T}=\vartheta_{b}^{T} \quad \vartheta^{G}=\vartheta_{b}^{G}
$$

- for $y=L$

$$
\frac{d \bar{u}}{d y}=0 \quad \frac{d \bar{\nu}}{d y}=0 \quad \frac{d \vartheta^{T}}{d y}=a_{T} \quad \frac{d \vartheta^{G}}{d y}=a_{G} \quad \bar{t}_{y y}=\bar{t}_{b}
$$

with the superscript $b$ denoting the boundary values.

\subsection{Nondimensionalisation}

With the dimensionless parameters defined in Table 2, in which $V_{0}$ is the characteristic velocity of the flow, equations (3.5) are recast in dimensionless forms

$$
\begin{aligned}
& 0=\frac{d}{d \tilde{y}}\left\{\frac{1-\bar{\nu}_{m} \tilde{\nu}_{s}}{1-\bar{\nu}_{m} \tilde{\nu}} \Xi_{1}+\frac{\tilde{\mathcal{F}}_{c}}{\left(\tilde{\nu}_{\infty}-\tilde{\nu}\right)^{8}}\left(\frac{d \tilde{u}}{d \tilde{y}}\right)^{2}\right\}+S_{1} \tilde{\nu} \sin \theta \\
& 0=\frac{d \tilde{\pi}}{d \tilde{y}}+S_{2} \tilde{\nu} \cos \theta \\
& 0=\frac{d}{d \tilde{y}}\left\{\frac{2 \tilde{\nu} \tilde{\mathcal{F}}_{c}}{\left(\tilde{\nu}_{\infty}-\tilde{\nu}\right)^{2}} \frac{d \tilde{\nu}}{d \tilde{y}}\right\}+\frac{1}{\tilde{\nu}}\left\{\tilde{\pi}+\frac{1-\bar{\nu}_{m} \tilde{\nu}_{s}}{1-\bar{\nu}_{m} \tilde{\nu}} \Xi_{2}-2 \tilde{\nu}^{2}(\tilde{\nu}-1)-\frac{2 \tilde{\nu} \tilde{\nu}_{\infty} \tilde{\mathcal{F}}_{c}}{\left(\tilde{\nu}_{\infty}-\tilde{\nu}\right)^{3}}\left(\frac{d \tilde{\nu}}{d \tilde{y}}\right)^{2}\right\} \\
& 0=\frac{\chi_{1}\left(\tilde{\mathcal{F}}_{c}-1\right)}{\left(\tilde{\nu}_{\infty}-\tilde{\nu}\right)^{8}}\left(\frac{d \tilde{u}}{d \tilde{y}}\right)^{3}-\frac{d^{2} \tilde{\vartheta}^{T}}{d \tilde{y}^{2}}-\chi_{2}\left(\frac{d \tilde{\vartheta}^{T}}{d \tilde{y}}\right)^{2} \\
& 0=-\frac{d^{2} \tilde{\vartheta}^{G}}{d \tilde{y}^{2}}-\chi_{3}\left(\frac{d \tilde{\vartheta}^{G}}{d \tilde{y}}\right)^{2}
\end{aligned}
$$

for $\tilde{u}(\tilde{y}), \tilde{\nu}(\tilde{y}), \tilde{\pi}(\tilde{y}), \tilde{\vartheta}^{T}(\tilde{y})$ and $\tilde{\vartheta}^{G}(\tilde{y})$, with $\tilde{\mathcal{F}}_{c}=1+\tilde{\vartheta}^{T}+\tilde{\vartheta}^{G}+\left(\tilde{\vartheta}^{T}\right)^{2}+\left(\tilde{\vartheta}^{G}\right)^{2}$, and the dimensionless BCs:

- for $\tilde{y}=0$

$$
\tilde{u}=0 \quad \tilde{\nu}=\tilde{\nu}_{b} \quad \tilde{\vartheta}^{T}=\tilde{\vartheta}_{b}^{T} \quad \tilde{\vartheta}^{G}=\tilde{\vartheta}_{b}^{G}
$$

- for $\tilde{y}=\tilde{L}$

$$
\frac{d \tilde{u}}{d \tilde{y}}=0 \quad \frac{d \tilde{\nu}}{d \tilde{y}}=0 \quad \frac{d \tilde{\vartheta}^{T}}{d \tilde{y}}=\tilde{a}_{T} \quad \frac{d \tilde{\vartheta}^{G}}{d \tilde{y}}=\tilde{a}_{G} \quad \tilde{\pi}_{b}=\tilde{\pi}_{b}
$$


Table 2. Dimensionless parameters

$$
\begin{array}{|lcccc|}
\xi^{2}=\frac{\alpha_{0}}{\beta_{0}}=\frac{1}{\ell^{2}} & \tilde{y}=\xi y & \tilde{L}=\xi L & \tilde{\nu}=\frac{\bar{\nu}}{\bar{\nu}_{m}} & \tilde{u}=\frac{\bar{u}}{V_{0}} \\
\tilde{\nu}_{\infty}=\frac{\bar{\nu}_{\infty}}{\bar{\nu}_{m}} & \tilde{n u}_{b}=\frac{\bar{\nu}_{b}}{\bar{\nu}_{m}} & \tilde{\vartheta}^{T}=\frac{\vartheta^{T}}{\vartheta^{M}} & \tilde{\vartheta}^{G}=\frac{\vartheta^{G}}{\vartheta^{M}} & \\
\Xi_{1}=\frac{\zeta_{2} \bar{Z}_{x y}+\zeta_{3} \bar{Z}_{x y}^{2}}{\mu_{0} \bar{\gamma}^{2} V_{0}^{2} \xi^{2}} & \Xi_{2}=\frac{\zeta_{1}+\zeta_{2} \bar{Z}_{y y}+\zeta_{3} \bar{Z}_{y y}^{2}}{\alpha_{0} \bar{\gamma} \bar{\nu}_{m}^{3}} & S_{1}=\frac{\bar{\nu}_{m} b}{\mu_{0} \bar{\gamma} V_{0}^{2} \xi^{3}} \\
S_{2}=\frac{b}{\alpha_{0} \bar{\nu}_{m}^{2} \xi} & \chi_{1}=\frac{\mu_{0} \bar{\gamma} V_{0}^{3} \xi}{f_{7} \vartheta^{M}} & \chi_{2}=\frac{f_{3} \vartheta^{M}}{f_{7}} & \chi_{3}=\frac{f_{6} \vartheta^{M}}{f_{8}} \\
\tilde{\pi}=\frac{-\bar{t}_{y y}}{\alpha_{0} \bar{\gamma}_{m}^{3}} & \tilde{\nu}_{s}=\frac{\bar{\nu}_{s}}{\bar{\nu}_{m}} & \tilde{\pi}_{b}=\frac{-\bar{t}_{b}}{\alpha_{0} \bar{\gamma} \bar{\nu}_{m}^{3}} & \tilde{\vartheta}_{b}^{T}=\frac{\vartheta_{b}^{T}}{\vartheta^{M}} \\
\tilde{\vartheta}_{b}=\frac{\vartheta_{b}^{G}}{\vartheta^{M}} & \tilde{a}_{T}=\frac{a_{T}}{\vartheta^{M} \xi} & \tilde{a}_{G}=\frac{a_{G}}{\vartheta^{M} \xi} & \\
\hline
\end{array}
$$

Equations (3.8)-(3.10) define a nonlinear BVP, with $\tilde{L}$ being the effect of flow thickness; $S_{2}$ the effect of gravity; $S_{1}$ the influence of viscosity for fixed $S_{2} ; \Xi_{1}$ and $\Xi_{2}$ the hypoplastic effect; $\chi_{1}$ the relative contribution between viscosity and turbulent kinetic energy flux; $\chi_{2}$ and $\chi_{3}$ the relative significances between two-fold turbulent kinetic energy fluxes and dissipations. For implementation of numerical simulation, the values of $\bar{\nu}_{m}, \bar{\nu}_{b}, \bar{\nu}_{\infty}$ and $\bar{\nu}_{s}$ are given by $\bar{\nu}_{b}=0.51$, $\bar{\nu}_{m}=0.555, \bar{\nu}_{\infty}=0.644, \bar{\nu}_{s}=0.25$ (thus, $\tilde{\nu}_{b}=0.919, \tilde{\nu}_{\infty}=1.16, \tilde{\nu}_{s}=0.451$ ), with fixed values of $\vartheta_{b}^{T}, \vartheta_{b}^{G}$ and $\bar{t}_{b}$ assumed as a first approximation (Bauer and Herle, 2000; Fang and Wu, 2014; Savage, 1993; Wang and Hutter, 1999).

Two-point nonlinear BVP (3.8)-(3.10) is solved numerically by using the iterative methods with a successive under-relaxation scheme (Fang and Wu, 2014; Wang and Hutter, 1999; Wendt, 2009). So, sequences of the primitive mean fields are calculated at each iteration step, which are incorporated into the next step, until demanded convergence is reached. Moreover, integrating last Eq. (3.8) yields an analytical solution of $\tilde{\vartheta}^{G}(\tilde{y})$ under a fixed value of $\chi_{3}$, viz., $\tilde{\vartheta}^{G}=\tilde{\vartheta}_{b}^{G}+$ $\chi_{3}^{-1} \ln \left(\left(1-\tilde{a}_{G} \chi_{3}(\tilde{L}-\tilde{y})\right) /\left(1-\tilde{a}_{G} \chi_{3} \tilde{L}\right)\right)$, indicating that $\tilde{\vartheta} G$ increases logarithmically from the plane toward the free surface, and corresponding to the previous works (Fang, 2009, 2016b; Fang and $\mathrm{Wu}, 2014)$.

\subsection{Numerical results}

Numerical tests show that only the relative magnitudes of the $\tilde{\nu}_{-}, \tilde{u}^{-}, \tilde{\vartheta}^{T}$ - and $\vartheta^{G}$-profiles are influenced by the variations in $S_{1}$ and $\chi_{1-3}$, but the tendencies remain unchanged. Thus, $S_{1}=0.02$ and $\chi_{1}=\chi_{2}=\chi_{3}=0.01$ are used, with $\tilde{\vartheta}_{b}^{T}=\tilde{\vartheta}_{b}^{G}=0.1$ for finite turbulent kinetic energies and dissipations on the boundary (Pudasaini and Hutter, 2007; Rao and Nott, 2008). Since $\Xi_{1}$ and $\Xi_{2}$ are of equal importance (Fang, 2009; Kirchner and Teufel, 2002), they are set equal. In all figures, the normalized calculated values are displayed for comparison.

Figure 2 illustrates the profiles of $\tilde{\nu}, \tilde{u}, \bar{\gamma} \tilde{\nu} s, \bar{\gamma} \tilde{\nu} k, \bar{\gamma} \tilde{\nu} H$ and $\bar{\gamma} \tilde{\nu} \varepsilon$ for variations in $\tilde{L}=[10,15,20]$ indicated by the arrows, with $\tilde{a}_{T}=\tilde{a}_{G}=0.1, \Xi_{1}=\Xi_{2}=0.01, \tilde{\pi}_{b}=0.01$ and $S_{2}=0.02$. The solid lines are the simulated results; the dashed lines are the laminar flow solutions from Fang (2009); the dotted line are Newtonian fluid characteristics in a laminar flow. Increasing $\tilde{L}$ tends to enlarge the difference in $\tilde{\nu}$ between the free surface and plane, as shown in Fig. 2a. This results from the weight of the granular body: when the flow is thicker, larger compressive stress applies on the grains in the thin layer immediately above the plane (the turbulent boundary layer, $T B L$ ), with maximum shearing there, causing the grains to collide intensively with one another and resulting in smaller values of $\tilde{\nu}$. Above this thin layer, there exists a relatively thick layer (the passive layer, $P L)$, in which the grains form a kind of inelastic network and behave as a lump 

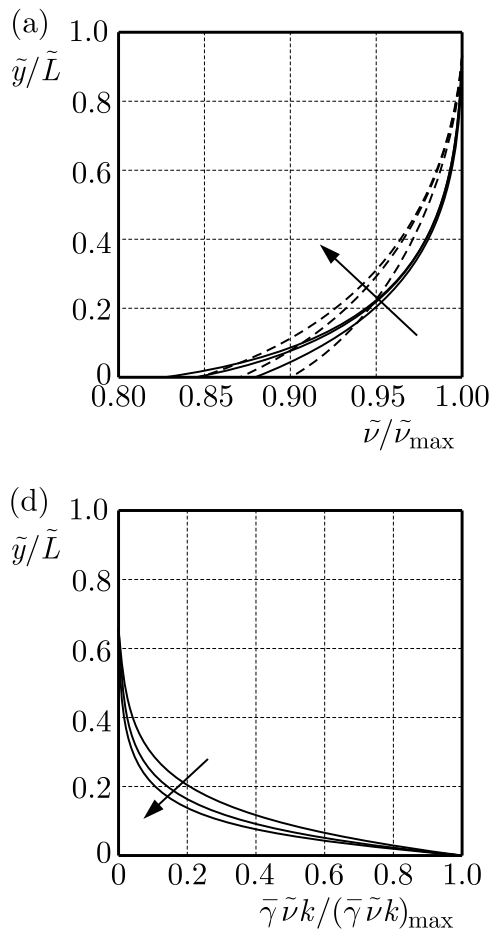
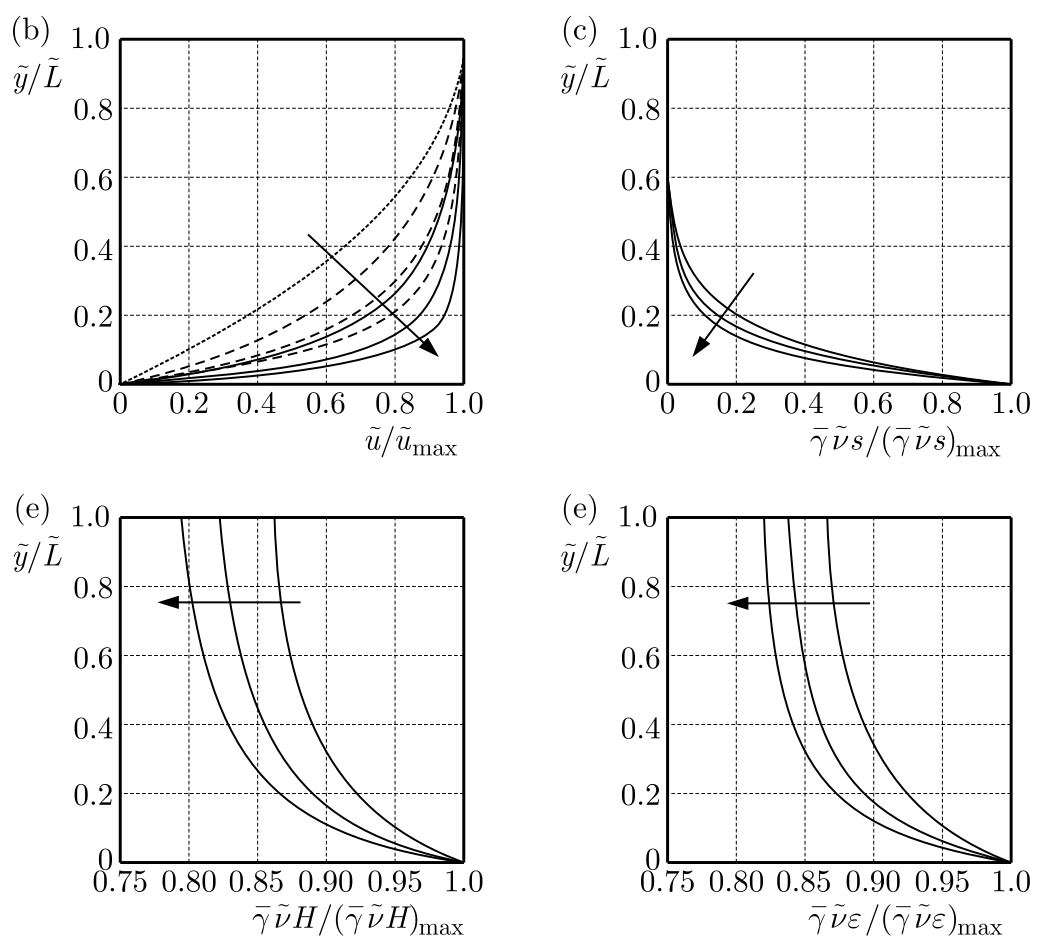

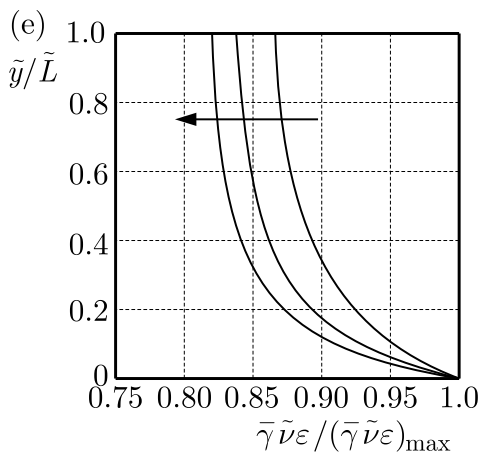

Fig. 2. Normalized profiles of $\tilde{\nu}, \tilde{u}, \bar{\gamma} \tilde{\nu} s, \bar{\gamma} \tilde{\nu} k, \bar{\gamma} \tilde{\nu} H, \bar{\gamma} \tilde{\nu} \varepsilon$, with $\tilde{L}=[10,15,20]$ indicated by the arrows. Dashed lines: laminar flow solutions; dotted line: laminar Newtonian flow

solid with nearly uniform $\tilde{\nu}$ and $\tilde{u}$, as displayed in Figs. 2a and 2b. As $\tilde{L}$ increases, the TBL becomes thinner with larger $\tilde{u}$-gradients at the interface between two layers. When compared with laminar flow solutions, the $\tilde{\nu}$ - and $\tilde{u}$-profiles are more convex, with larger amplitudes in the PL. These are due to the influence of turbulent kinetic energy and dissipation, to be discussed later.

Two-fold turbulent kinetic energies in Figs. 2c and $2 \mathrm{~d}$ decrease from their maximum values in the plane toward nearly vanishing values on the free surface exponentially. Similar tendencies appear for the profiles of $\bar{\gamma} \tilde{\nu} H$ and $\bar{\gamma} \tilde{\nu} \varepsilon$ in Figs. 2e and 2f, except for finite values on the free surface. As $\tilde{L}$ increases, $\bar{\gamma} \tilde{\nu} s, \bar{\gamma} \tilde{\nu} k, \bar{\gamma} \tilde{\nu} H$ and $\bar{\gamma} \tilde{\nu} \varepsilon$ decrease more obviously. These correspond not only to those of Newtonian fluids in turbulent boundary layer flows, but also are justified, for turbulent kinetic energy and dissipation should assume maximum values in the regions where shearing is maximum, and a larger turbulent kinetic energy induces larger turbulent dissipation (Batchelor, 1993; Tsinober, 2009). Although in Newtonian fluids and dry granular avalanches the turbulent kinetic energies and dissipations evolve in a similar manner, their vanishing values on the free surface are identified for Newtonian fluids, while it is not so for dry granular avalanches. These reflect the discrete nature of dry granular systems.

Although the TBL and PL can be identified by the profiles of $\nu$ and $u$ in laminar formulations (e.g. Fang, 2009; Wang and Hutter, 1999), they are preferably recognized by the distributions of $\bar{\gamma} \tilde{\nu} s, \bar{\gamma} \tilde{\nu} k, \bar{\gamma} \tilde{\nu} H$ and $\bar{\gamma} \tilde{\nu} \varepsilon$. In the PL, the dominant grain-grain interaction is the long-term one, causing the grains to form a kind of inelastic network to yield nearly vanishing $\bar{\gamma} \tilde{\nu} s$ and $\bar{\gamma} \tilde{\nu} k$, and finite $\bar{\gamma} \tilde{\nu} H$ and $\bar{\gamma} \tilde{\nu} \varepsilon$. On the other hand, the grains in the TBL are dominated by the short-term interaction, giving a rise to intensive turbulent fluctuation with significant $\bar{\gamma} \tilde{\nu} s, \bar{\gamma} \tilde{\nu} k$, $\bar{\gamma} \tilde{\nu} H$ and $\bar{\gamma} \tilde{\nu} \varepsilon$, resulting in larger $\tilde{\nu}$ and $\tilde{u}$ in the PL, when compared with laminar flow solutions.

Figure 3 illustrates the profiles of $\tilde{\nu}, \tilde{u}, \bar{\gamma} \tilde{\nu} s, \bar{\gamma} \tilde{\nu} k, \bar{\gamma} \tilde{\nu} H$ and $\bar{\gamma} \tilde{\nu} \varepsilon$ for variations in $S_{2}=[0.01,0.035,0.07]$ indicated by the arrows, with $\tilde{a}_{T}=\tilde{a}_{G}=0.1, \bar{L}=15, \Xi_{1}=\Xi_{2}=0.01$ and $\tilde{\pi}_{b}=0.01$. Increasing $S_{2}$ tends to enhance the gravitational effect, resulting in more convex $\tilde{\nu}$ - and $\tilde{u}$-profiles in Figs. 3a and 3b. This goes back to the influence of a larger grain weight. As 

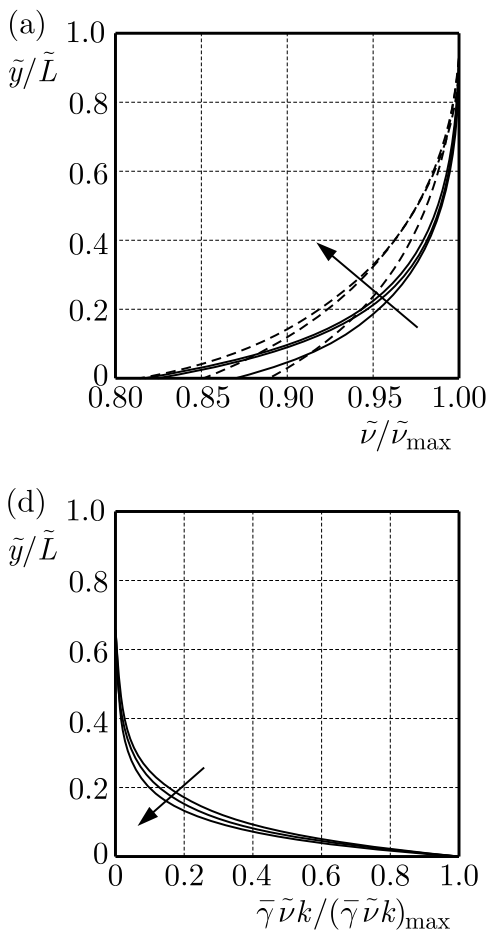
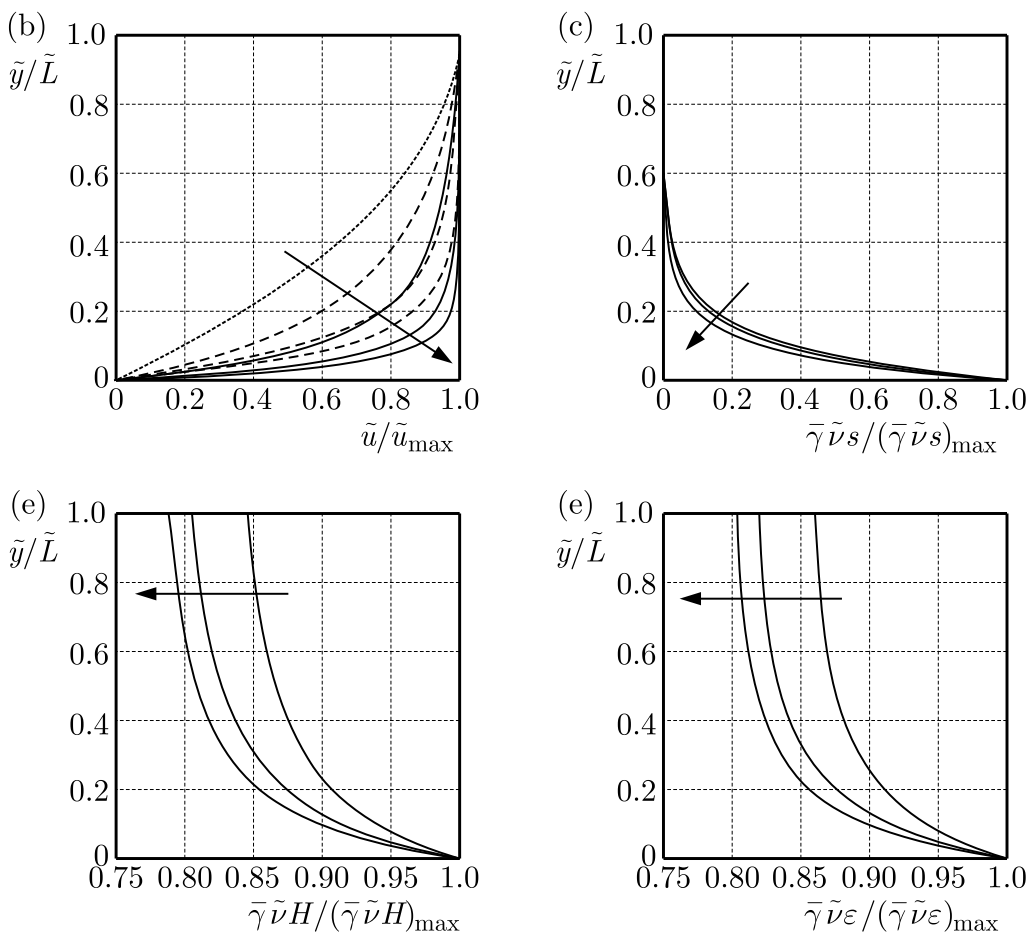

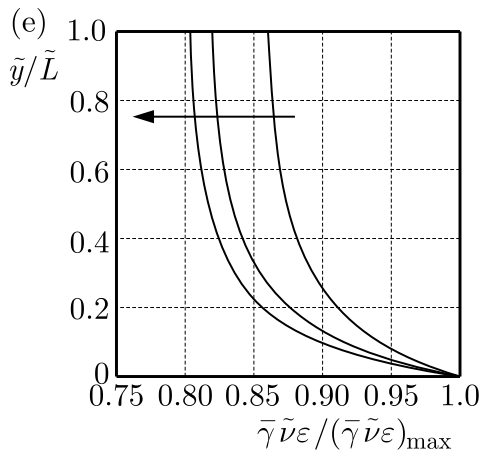

Fig. 3. Normalized profiles of $\tilde{\nu}, \tilde{u}, \bar{\gamma} \tilde{\nu} s, \bar{\gamma} \tilde{\nu} k, \bar{\gamma} \tilde{\nu} H, \bar{\gamma} \tilde{\nu} \varepsilon$, with $S_{2}=[0.01,0.035,0.07]$ indicated by the arrows. Dashed lines: laminar flow solutions; dotted line: laminar Newtonian flow

$S_{2}$ increases, most $\bar{\gamma} \tilde{\nu} s, \bar{\gamma} \tilde{\nu} k, \bar{\gamma} \tilde{\nu} H$ and $\bar{\gamma} \tilde{\nu} \varepsilon$ are confined within even thinner TBLs, resulting in more energetic grain collisions there, as shown in Figs. 3c-3f. In addition, $\bar{\gamma} \tilde{\nu} s, \bar{\gamma} \tilde{\nu} k, \bar{\gamma} \tilde{\nu} H$ and $\bar{\gamma} \tilde{\nu} \varepsilon$ evolve with similar tendencies described in Fig. 2. Due to the distributions of two-fold turbulent kinetic energies, the $\tilde{\nu}$ - and $\tilde{u}$-profiles are more convex than their laminar-flow counterparts.

The influence of $\tilde{\pi}_{b}$ is summarized in Fig. 4, with $\tilde{a}_{T}=\tilde{a}_{G}=0.1, \Xi_{1}=\Xi_{2}=0.01, \tilde{L}=15$, $S_{2}=0.02$ and $\tilde{\pi}_{b}=[0.01,0.1,0.25]$ indicated by the arrows. Increasing $\tilde{\pi}_{b}$ is to apply larger normal traction on the free surface, exciting the grains in the TBL to collide with one another more vigorously. This reduces base friction, resulting in more convex $\tilde{\nu}$ - and $\bar{u}$-profiles in Figs. $4 \mathrm{a}$ and $4 \mathrm{~b}$, and thicker PLs. Figures 4c-4f show that as $\tilde{\pi}_{b}$ increases, $\bar{\gamma} \tilde{\nu} s, \bar{\gamma} \tilde{\nu} k, \bar{\gamma} \tilde{\nu} H$ and $\bar{\gamma} \tilde{\nu} \varepsilon$ are confined mostly in the TBL and decrease exponentially from the plane toward the free surface. The profiles of $\tilde{\nu}$ and $\tilde{u}$ are more convex than their laminar flow counterparts due to the influences of $\bar{\gamma} \tilde{\nu} s, \bar{\gamma} \tilde{\nu} k, \bar{\gamma} \tilde{\nu} H$ and $\bar{\gamma} \tilde{\nu} \varepsilon$.

Simulations for variations in $\Xi_{1}$ and $\Xi_{2}$ are summarized in Fig. 5, with $\Xi_{1}=\Xi_{2}=$ $[0.01,0.05,0.1]$ indicated by the arrows; $\tilde{a}_{T}=\tilde{a}_{G}=0.1, \tilde{\pi}_{b}=0.01, \tilde{L}=15$ and $S_{2}=0.02$. When $\Xi_{1}$ and $\Xi_{2}$ increase, the hypoplastic effect inside a granular RVE is enhanced, which weakens the frictional contact between the grains in the TBL, giving a rise to reduced $\bar{\gamma} \tilde{\nu} s, \bar{\gamma} \tilde{\nu} k$, $\bar{\gamma} \tilde{\nu} H$ and $\bar{\gamma} \tilde{\nu} \varepsilon$ with thinner TBLs. With an enhanced hypoplastic effect, most $\bar{\gamma} \tilde{\nu} s, \bar{\gamma} \tilde{\nu} k, \bar{\gamma} \tilde{\nu} H$ and $\bar{\gamma} \tilde{\nu} \varepsilon$ are confined within even thinner TBLs, which are equally recognized in the $\tilde{\nu}$ - and $\tilde{u}$-profiles.

Calculations for variations in $\tilde{a}_{T}$ and $\tilde{a}_{G}$ are given in Fig. 6 , with $\tilde{a}_{T}=\tilde{a}_{G}=[0.01,0.025,0.05]$ indicated by the arrows; $\Xi_{1}=\Xi_{2}=0.01, \tilde{\pi}_{b}=0.01, S_{2}=0.02$ and $\tilde{L}=15$. Equal values of $\tilde{a}_{T}$ and $\tilde{a}_{G}$ are used for simplicity. Increasing $\tilde{a}_{T}$ and $\tilde{a}_{G}$ allows more fluxes of $\bar{\gamma} \tilde{\nu} s$ and $\bar{\gamma} \tilde{\nu} k$ enter into the granular body from the free surface, inducing more $\bar{\gamma} \tilde{\nu} H$ and $\bar{\gamma} \tilde{\nu} \varepsilon$ for counter-balance. This yields more thicker PLs, illustrated by more convex profiles of $\tilde{\nu}, \tilde{u}, \bar{\gamma} \tilde{\nu} s, \bar{\gamma} \tilde{\nu} k, \bar{\gamma} \tilde{\nu} H$ and $\bar{\gamma} \tilde{\nu} \varepsilon$. These correspond to field observations, for in the PL, the grains are interlocked and behave as a lump solid, causing $\bar{\gamma} \tilde{\nu} H$ and $\bar{\gamma} \tilde{\nu} \varepsilon$ to overcome $\bar{\gamma} \tilde{\nu} s$ and $\bar{\gamma} \tilde{\nu} k$. 
(a)

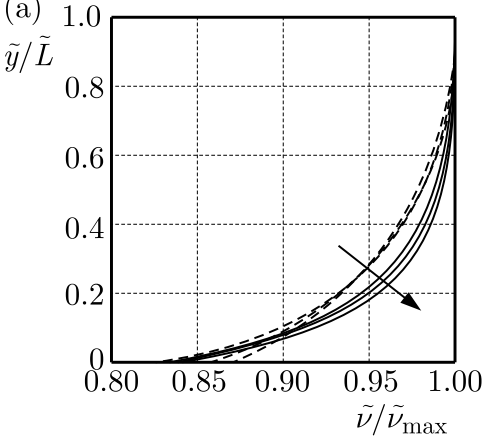

(d)

$\tilde{y} / \tilde{L}$

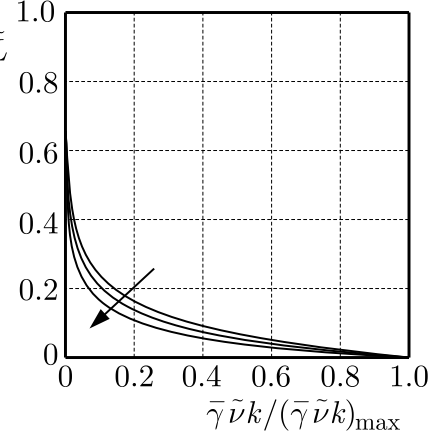

(b)

$\tilde{y} / \tilde{L}$

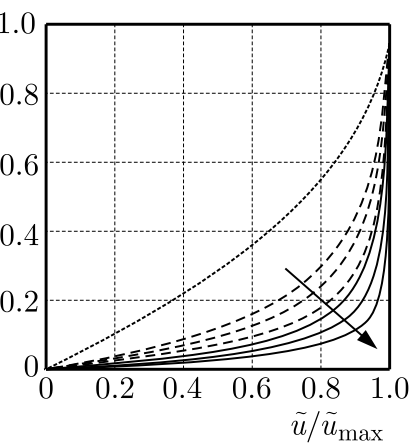

(e)

$\tilde{y} / \tilde{L}$

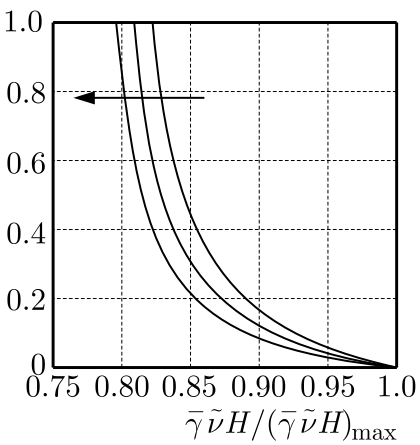

(c)

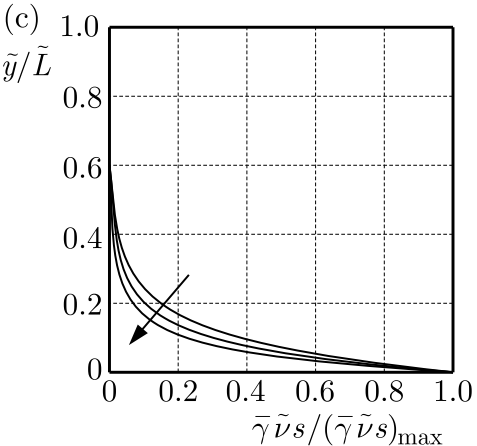

(e)

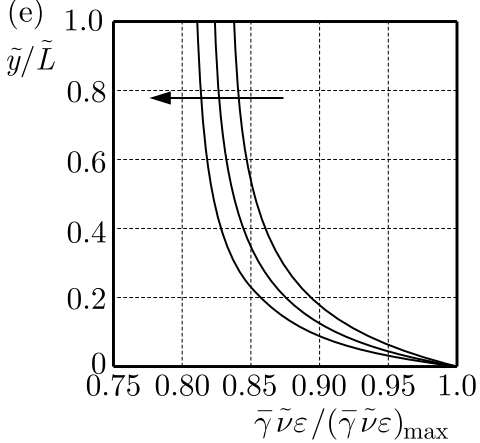

Fig. 4. Normalized profiles of $\tilde{\nu}, \tilde{u}, \bar{\gamma} \tilde{\nu} s, \bar{\gamma} \tilde{\nu} k, \bar{\gamma} \tilde{\nu} H, \bar{\gamma} \tilde{\nu} \varepsilon$, with $\tilde{\pi}_{b}=[0.01,0.1,0.25]$ indicated by the arrows. Dashed lines: laminar flow solutions; dotted line: laminar Newtonian flow
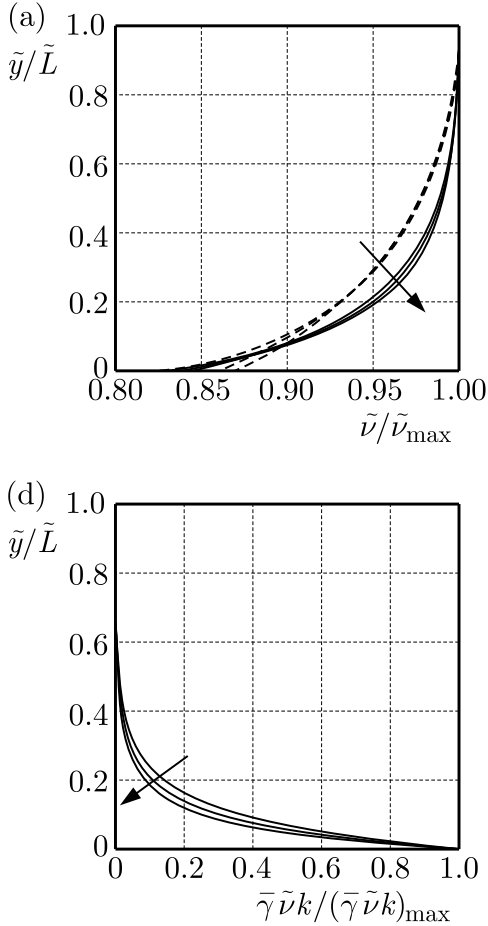

(b)

$\tilde{y} / \tilde{L}$

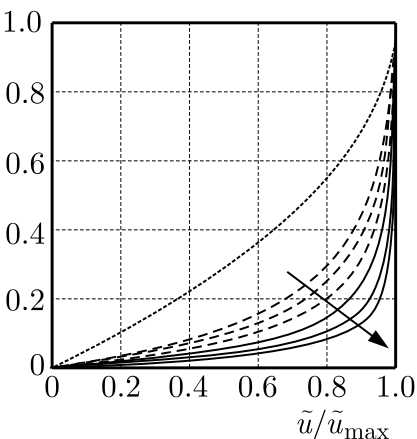

(e)

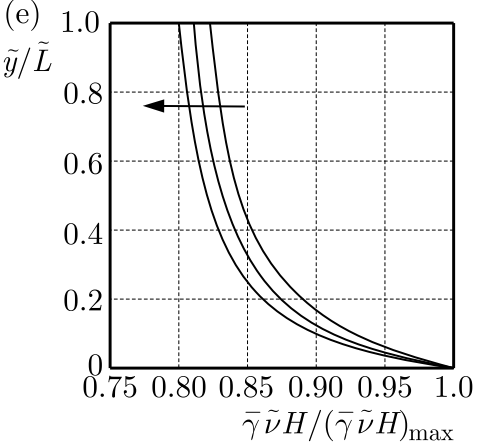

(c)

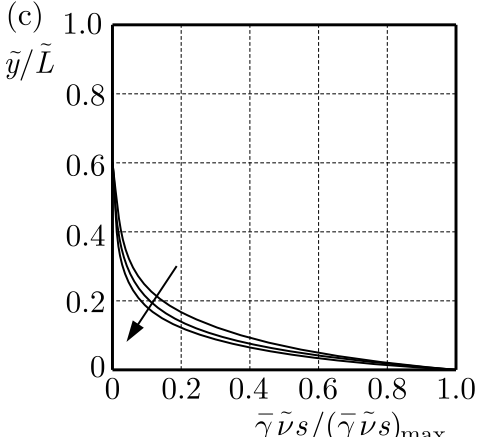

(e)

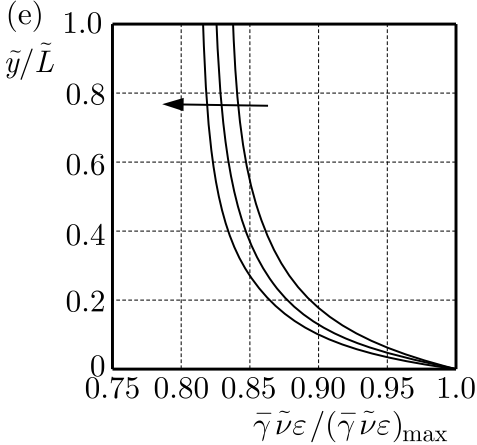

Fig. 5. Normalized profiles of $\tilde{\nu}, \tilde{u}, \bar{\gamma} \tilde{\nu} s, \bar{\gamma} \tilde{\nu} k, \bar{\gamma} \tilde{\nu} H, \bar{\gamma} \tilde{\nu} \varepsilon$, with $\Xi_{1}=\Xi_{2}=[0.01,0.5,0.1]$ indicated by the arrows. Dashed lines: laminar flow solutions; dotted line: laminar Newtonian flow 

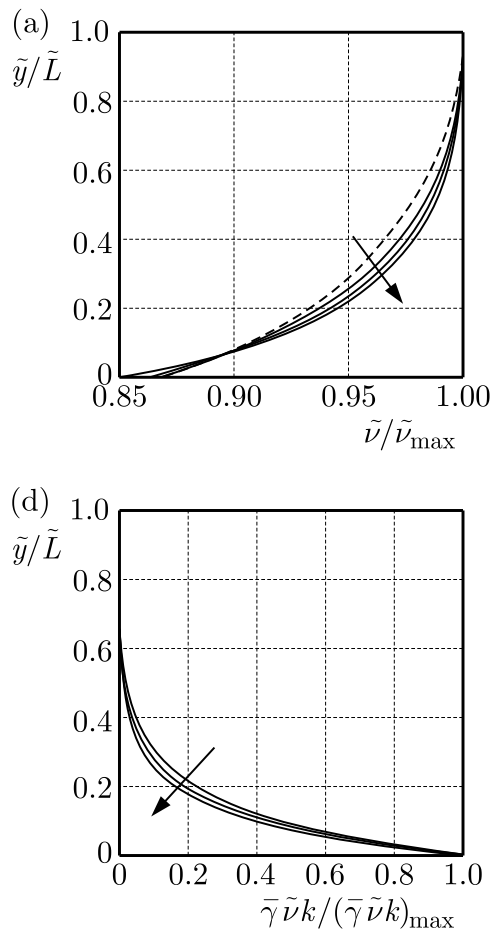
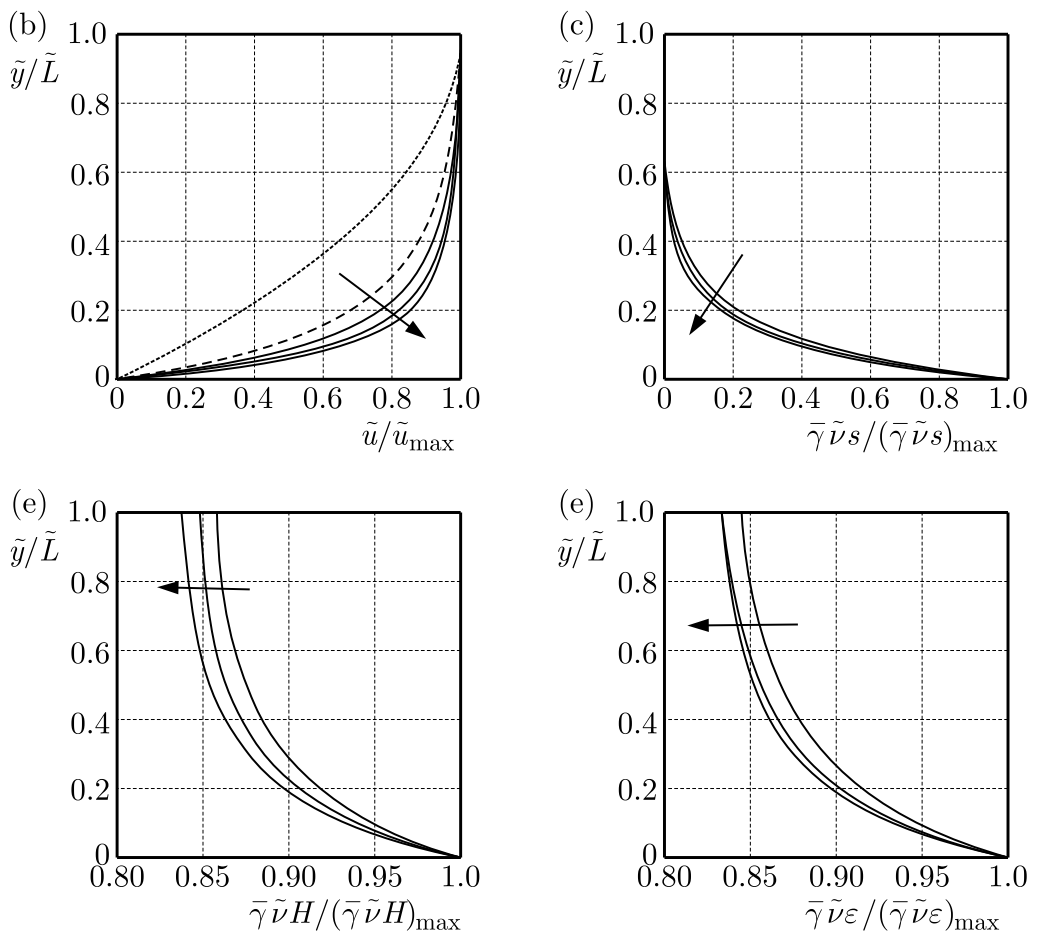

Fig. 6. Normalized profiles of $\tilde{\nu}, \tilde{u}, \bar{\gamma} \tilde{\nu} s, \bar{\gamma} \tilde{\nu} k, \bar{\gamma} \tilde{\nu} H, \bar{\gamma} \tilde{\nu} \varepsilon$, with $\tilde{a}_{T}=\tilde{a}_{G}=[0.01,0.025,0.05]$ indicated by the arrows. Dashed lines: laminar flow solutions; dotted line: laminar Newtonian flow

\section{Conclusions and discussions}

The derived equilibrium closure relations in Part I (Fang, 2016b) have been implemented to obtain a zero-order closure model, which has beem applied to analyses of a stationary avalanche down an incline; numerical simulations have been compared with laminar flow solutions.

While $\tilde{\nu}$ and $\tilde{u}$ evolve from their minimum values on the plane toward maximum values on the free surface, $\bar{\gamma} \tilde{\nu} s, \bar{\gamma} \tilde{\nu} k, \bar{\gamma} \tilde{\nu} H$ and $\bar{\gamma} \tilde{\nu} \varepsilon$ distribute in a reverse manner, with most of them confined within the TBL immediately above the plane. Above this, there exists a PL in which the grains behave as a lump solid with nearly uniform $\tilde{\nu}$ and $\tilde{u}$. In the TBL, the grains are dominated by the short-term interaction, giving a rise to intensive turbulent fluctuation with significant turbulent kinetic energy and dissipation, while those in the PL are dominated by the long-term interaction to form a kind of inelastic network. Two layers are preferable recognized from the turbulent kinetic energy and dissipation profiles.

The TBL and PL of a dry granular avalanche are similar to those of Newtonian fluids in turbulent boundary layer flows. Although the turbulent kinetic energies and dissipations evolve in a similar manner, their vanishing values on the free surface are found for Newtonian fluids, while nearly vanishing turbulent kinetic energies and finite turbulent dissipations are obtained for granular avalanches, resulted from their discrete nature and different dominant grain-grain interactions in the TBL and PL. Discrepancies in the estimated $\tilde{\nu}$ - and $\tilde{u}$-profiles from the laminar flow solutions suggest that the energy cascade induced by turbulent fluctuation needs to be considered for better estimations on the characteristics of dry granular avalanches.

\section{Acknowledgements}

The author is indebted to the Ministry of Science and Technology, Taiwan, for the financial support through the project MOST 103-2221-E-006-116-. The author also thanks the editor and Professor Janusz Badur for the detailed comments and suggestions which led to improvements. 


\section{References}

1. Ai J., Langston P.A., Yu H.-S., 2014, Discrete element modeling of material non-coaxiality in simple shear flows, International Journal for Numerical and Analytical Methods in Geomechanics, 38, 6, 615-635

2. Batchelor G.K., 1993, The Theory of Homogeneous Turbulence, Cambridge University Press, Cambridge New York

3. Bauer E., Herle I., 2000, Stationary states in hypoplasticity, [In:] Constitutive Modeling of Granular Materials, Kolymbas D. (ed.), Springer Verlag, Berlin, 167-192

4. Buscamera G., 2014, Uniqueness and existence in plastic models for unsaturated soils, Acta Geotechnica, 9, 313-327

5. FAnG C., 2008, Modeling dry granular mass flows as elasto-visco-hypoplastic continua with microstructural effects. II. Numerical simulations of benchmark flow problems, Acta Mechanica, 197, 191-209

6. FAnG C., 2009, Gravity-driven dry granular slow flows down an inclined moving plane: a comparative study between two concepts of the evolution of porosity, Rheologica Acta, 48, 971-992

7. FAnG C., 2016a, A $k-\varepsilon$ turbulent closure model of an isothermal dry granular dense matter, Continuum Mechanics and Thermodynamics, 28, 4, 1048-1069

8. FANG C., 2016b, On the turbulent boundary layer of a dry granular avalanche down an incline. I. Thermodynamic analysis, Journal of Theoretical and Applied Mechanics, 54, 3, 1051-1062

9. FANG C., WU W., 2014, On the weak turbulent motions of an isothermal dry granular dense flow with incompressible grains. Part II. Complete closure models and numerical simulations, Acta Geotechnica, 9, 739-752

10. Fellin W., 2013, Extension to barodesy to model void ratio and stress dependency of the $\mathrm{K}_{0}$ value, Acta Geotechnica, 8, 561-565

11. Fuentes W., Triantaftllidis T., Lizcano A., 2012, Hypoplastic model for sands with loading surface, Acta Geotechnica, 7, 177-192

12. Herle I., Gudehus G., 1999, Determination of parameters of a hypoplastic constitutive model from properties of grain assemblies, Mechanics of Cohesive and Frictional Materials, 4, 461-485

13. Kirchner N., Teufel A., 2002, Thermodynamically consistent modeling of abrasive granular materials. II: Thermodynamic equilibrium and applications to steady shear flows, Proceeding of Royal Society London A, 458, 3053-3077

14. Marcher Th., Vermeer P.A., Wolffersdorf P.-A., 2000, Hypoplastic and elastoplastic modeling - a comparison with test data, [In:] Constitutive Modeling of Granular Materials, Kolymbas D. (ed.), Springer Verlag, Berlin, 353-371

15. Niemunis A., Grandas-Tavera C.E., Prada-Sarmiento L.F., 2009, Anisotropic viscohypoplasticity, Acta Geotechnica, 4, 293-314

16. Pudasaini S., Hutter K., 2007, Avalanche Dynamics, Springer Verlag, Berlin Heidelberg

17. Rao K.K., Nott P.R., 2008, Introduction to Granular Flows, Cambridge University Press, London New York

18. Richman M.W., Marciniec R.P., 1990, Gravity-driven granular flows of smooth, inelastic spheres down bumpy inclines, Journal of Applied Mechanics, 57, 1036-1043

19. Savage S.B., 1993, Mechanics of granular flows, [In:] Continuum Mechanics in Environmental Sciences and Geophysics, Hutter K. (ed.), Heidelberg, Springer, 467-522

20. Tsinober A., 2009, An Informal Conceptual Introduction to Turbulence, Springer, Heidelberg

21. WAng Y., Hutter K., 1999, A constitutive theory of fluid-saturated granular materials and its application in gravitational flows, Rheologica Acta, 38, 214-223

22. Wang Y., Hutter K., 2001, Granular material theories revisited, [In:] Geomorphological Fluid Mechanics, Balmforth N.J., Provenzale A. (eds.), Heidelberg, Springer, 79-107

23. Wendt J.F., 2009, Computational Fluid Dynamics: An Introduction, Springer, Berlin Heidelberg 